\title{
CLADOSPORIUM HERBARUM LINK, AGENT D'ACCIDENTS TARDIFS DE «BLEU » SUR LES FROMAGES A PATE MOLLE, ET SPECIALEMENT LE CAMEMBERT
}

\author{
par \\ J. JACQUET et M. DESFLEURS
}

\section{I. - Introduction}

Nous avons eu déjà l'occasion de souligner, à plusieurs reprises, que les fromagers confondaient, sous le nom de "bleu ", différents champignons dont le seul caractère commun était justement d'avoir des conidiospores colorés de la même façon [9]. L'origine des espèces en cause, la gravité des accidents produits, les moyens à mettre en œuvre pour éviter une invasion future ou guérir celle qui est en cours, ne sont pas du tout les mêmes, d'où l'intérêt de bien préciser chaque fois la souche à laquelle on a vraiment affaire. C'est la raison pour laquelle l'un d'entre nous avait, autrefois, préconisé la constitution d'un Centre de Recherches de Microbiologie laitière, doté au moins d'un spécialiste de l'étude des champignons qui intéressent les professionnels. Il aurait dû y être constitué une collection susceptible d'aider tous ceux qui auraient à se pencher sur ce problème. La sélection de souches à tel ou tel caractère physiologique dominant ou intéressant, ainsi que la recherche des ferments lactiques normands aurait été une autre branche d'activité de ce Centre. Il nous paraît toujours d'autant plus nécessaire que beaucoup d'industriels s'orientent vers la fabrication de camemberts pasteurisés et utilisent des levains fortement acidifiants, qui sont, en fait, destinés au beurre et, par surcroît, d'origine étrangère. Il est évident que l'étude des germes naturels devrait donner de bien meilleurs résultats. On ne sait pour quelle raison ce projet n'a pas intéressé les Commissions compétentes qui décident, dans la capitale, de l'orientation des recherches faites dans notre pays. Peut-être, les économistes ont-ils pensé que l'introduction annuelle de microbes étrangers dont la valeur totale est de l'ordre de $500000 \mathrm{~F}$ n'était pas catastrophique pour notre balance commerciale. C'est oublier que la question n'est pas seulement d'ordre financier, mais, qu'en dehors des sorties de devises, faibles il est vrai, il faut tenir compte, d'abord et surtout, d'une amélioration d'ensemble de qualité de tous les produits, qui est

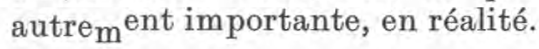


Indépendamment de l'étude des ferments, de l'isolement et de la sélection des germes naturels, l'étude des activités enzymatiques et de leurs conditions d'action s'impose. On devrait aboutir, même, à l'extraction de ces diastases, et tenter de remplacer certaines flores actuelles par les ferments solubles à l'aide desquelles les ferments figurés agissent. On obtiendrait, alors, des conditions très précises de fabrication et d'affinage. Tout ne peut, évidemment, être modifié, car on aura affaire parfois à des activités mixtes, telles celles du Penicillium caseicolum, par exemple, qui, à côté des dégradations aboutissant à l'affinage, brûle dans des combustions respiratoires l'acide lactique préalablement produit. Mais il est certain que c'est là un aspect moderne de la microbiologie fromagère qui doit être entrepris maintenant, et pour lequel un Centre spécialisé d'étude et de collection de souches microbiennes reste indispensable.

Par ailleurs, on n'utilise parmi les champignons que certaines variétés plus ou moins naturelles ou adaptées de quelques espèces. Il doit être sûrement avantageux d'en trouver d'autres, compte tenu de leurs divers effets, car des actions toxiques insoupçonnées peuvent parfois apparaître (comme avec Aspergillus flavus, par exemple).

Cependant, nous avons trouvé sur place un écho plus favorable auprès des dirigeants du Syndicat du Véritable Camembert de Normandie qui, pendant quelques années, nous ont apporté leur aide pour résoudre au moins un problème qui les préoccupait : l'invasion massive par des champignons bleus que l'on constatait alors, très fréquemment, et dans de nombreuses usines. Dès 1951 [8], puis en 1954 [9], nous avons établi que deux facteurs concomitants intervenaient pour expliquer cet envahissement :

a) La sélection, par les fabricants de levains, de souches strictement acidophiles de Penicillium caseicolum Bainier $(:=P$. candidum Link).

b) L'introduction, par les fabricants de sel, d'hydroxycarbonate de magnésium qui, en excès ou mal mélangé, alcalinisait la surface du camembert ensemencé, gênait ou empêchait la pousse de l'espèce normale et favorisait l'installation des souches de $P$. glaucum, causes de l'accident, qui, elles, ne sont pas limitées, ainsi, par le $p \mathrm{H}$.

L'application des règles que nous préconisâmes alors fit totalement disparaître ce type de maladies. On ne rencontre plus maintenant que des aspects différents, des installations, par petites taches, de bleu, sur lesquels nous comptons également nous expliquer et dont le mémoire actuel représente un des points particuliers.

Quelques années après nous, M. Menderez, en 1958 [16] et M. Masson, en 1959 [15], firent, à Nancy, des observations identiques et proposèrent l'emploi d'un sel acidifiant, par adjonction d'acide 
citrique ou de phosphate acide de calcium. L'un d'entre nous étudia, au laboratoire et en grande pratique industrielle, le sel ainsi préparé : il ne produit pas les effets compensateurs désirés, car intervient puissamment le pouvoir tampon du caillé de fromage [3].

A notre avis, le sel ne doit être ni acidifiant, ni alcalinisant et le talc nous paraissait, à cet égard, beaucoup mieux convenir comme substance enrobant les cristaux de chlorure de sodium en vue d'empêcher leur "hygroscopicité" [9]. Par ailleurs, les adjonctions proposées doivent, dans tous les cas, être longuement étudiées dans toutes leurs conséquences avant d'être généralisées, dans une industrie biologique comme celle des fromages où la moindre modification du milieu peut tout changer.

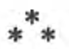

A notre connaissance, la présence de Cladosporium herbarum Link, sur les camemberts, n'a guère attiré l'attention puisqu'en 1955 , l'un et l'autre des auteurs de ce travail, et d'une façon indépendante, l'identifièrent pour la première fois dans deux usines différentes. Nos travaux sur le Penicillium glaucum et ses rapports avec la composition du sel étaient alors terminés; les graves accidents, spectaculaires et généralisés du bleu, avaient disparu. Il était donc naturel que les fromagers s'inquiétâssent de l'apparition d'une moisissure bleue sur leurs camemberts, non plus vers le début de la mise au haloir et qui envahissait alors tout, comme c'était précédemment le cas, mais plus tardivement, commençant vers le $9 \mathrm{e}-11^{\mathrm{e}}$ jour, et formant seulement quelques petits points. L'un de nous a tenté, par ailleurs, de dégager la "philosophie " des maladies des fromages et de démontrer le parallèle entre cette "pathologie" et celle, notamment, des animaux [7]. On peut dire que l'on retrouve, là encore, un fait bien connu des microbiologistes. Quand on possède des moyens de lutte efficaces contre des germes majeurs, responsables d'énormes accidents (staphylocoques, streptocoques, bacilles tuberculeux, par exemple) paraissent, alors, prendre plus d'importance qu'autrefois, des espèces mineures, peu pathogènes ou peu fréquentes, comme le bacille pyocyanique, dont le rôle était pour ainsi dire étouffé par les espèces plus puissantes. Il en est de même, pour les camemberts, du Cladosporium herbarum par rapport au Penicillium glaucum.

Aussi, ne pensâmes-nous point que le Cladosporium herbarum prenait la relève des Penicillium à spores bleues ( $P$. glaucum, $P$. brevicompactum, $P$. brunneoviolaceum) déjà observés. Nous croyons plutôt qu'il était tout simplement confondu dans ce vaste ensemble étiqueté d'une façon bien vague, "bleu " par les professionnels et représentait un cas relativement assez rare, mais intéressant. 


\section{II. - Classification}

Le Cladosporium herbarum Link (ex Fries) appartient au groupe des champignons dont on ne connaît pas encore le mode de reproduction parfait (et pour cela dénommés Fungi Imperfecti) et selon Saccardo [18 à 20] par exemple, à l'ordre des Hyphomycétales et dans celui-ci, à la famille des Dematiaceae (ou Dématiées) possédant un mycelium et des spores de couleur foncé.

Langeron [11] le classe dans les Adélomycètes, ordre assez assimilable aux Hyphomycètes et à l'intérieur de ceux-ci, dans la tribu des Thallosporées, espèces qui se reproduisent, d'une façon asexuée, en donnant des spores au dépens du thalle préexistant, des thallospores. Cette dernière notion est, cependant, discutée, comme nous le verrons plus loin.

Par ailleurs, $C l$. herbarum est un champignon des fumagines qui forme un enduit noir bistré, charbonneux, à la surface de nombreuses feuilles, si l'état hygrométrique est suffisamment élevé et si des substances sucrées (de la plante ou de secrétions d'insectes) y sont présentes. Ils peuvent avoir, enfin, un thalle dit fumagoilde, dissocié, constitué par des éléments arrondis, isolés ou groupés, à membrane épaisse, de couleur fuligineuse, plus volumineuse que les éléments normaux et qui se divisent par des cloisons diamétrales ou méridiennes, constituant quatre portions qui s'arrondissent ensuite (fig. 1).

Le mode de reproduction des Cladosporium est diversement interprété : certains y voient l'apparition d'un appareil conidien dressé perpendiculairement au thalle végétatif (Saccardo), d'autres pensent qu'il s'agit simplement de spores dérivant du thalle (Masson, Langeron), qui suivant leur forme, quadrangulaires, seraient des arthrospores, ou ovalaires, des blastospores.

Dans le premier cas, on fera de l'espèce une conidiosporée, dans l'autre cas, une thallosporée.

De toutes façons, il est certain que les spores poussent les unes sur les autres; les plus anciennes, celles qui sont le plus près du mycélium, sont plus longues et souvent séparées en deux par cloisonnement. Les conidies plus récentes sont plus petites et non divisées.

Primitivement, certains auteurs réservaient le nom de Cladosporium aux souches à spores cloisonnées et baptisaient Hormodendron celles à spores monocellulaires. Or, les deux types de spores coexistent toujours dans la même culture, comme le montre très bien la microscopie électronique (fig. 3), et leur forme, leur taille dépendent de leur position dans la chaîne conidienne. On aurait, alors affaire à une simple synonymie, comme le pensent Brookes et Hansford qui, après avoir étudié un certain nombre de souches, pensent qu'elles se rattachent toutes à l'espèce Cladosporium 


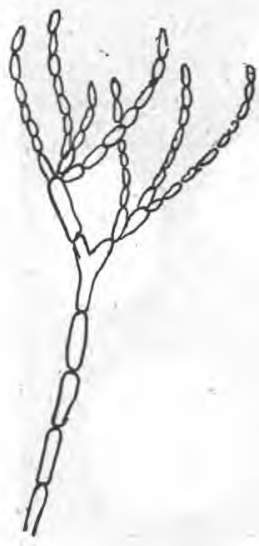

a
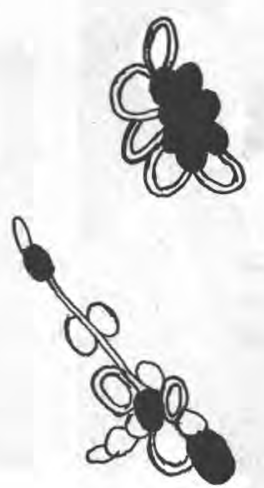

b

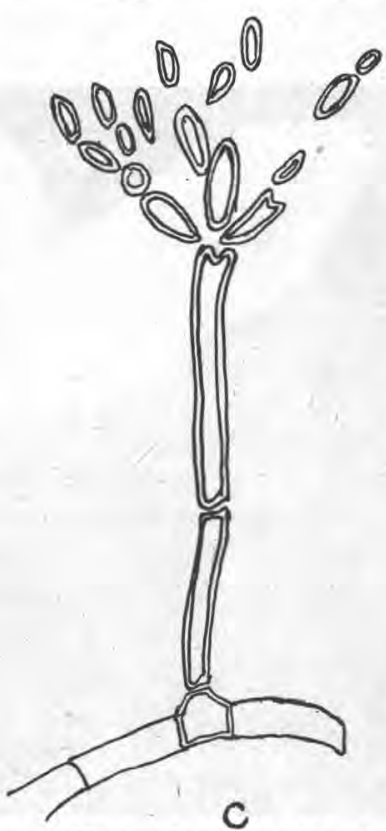

FIG. 1. - Aspect schématique des Cladosporium et Hormodendron, d'après Langeron et Planchon.

a) Appareil conidien, les conidiospores pouvant être interprétées comme dérivant du thalle. $b$ ) Formes fumagoïdes en milieu liquide (culture en milieu de Raulin).c) Forme vigoureuse, décrite sous le nom de Hormodendron.

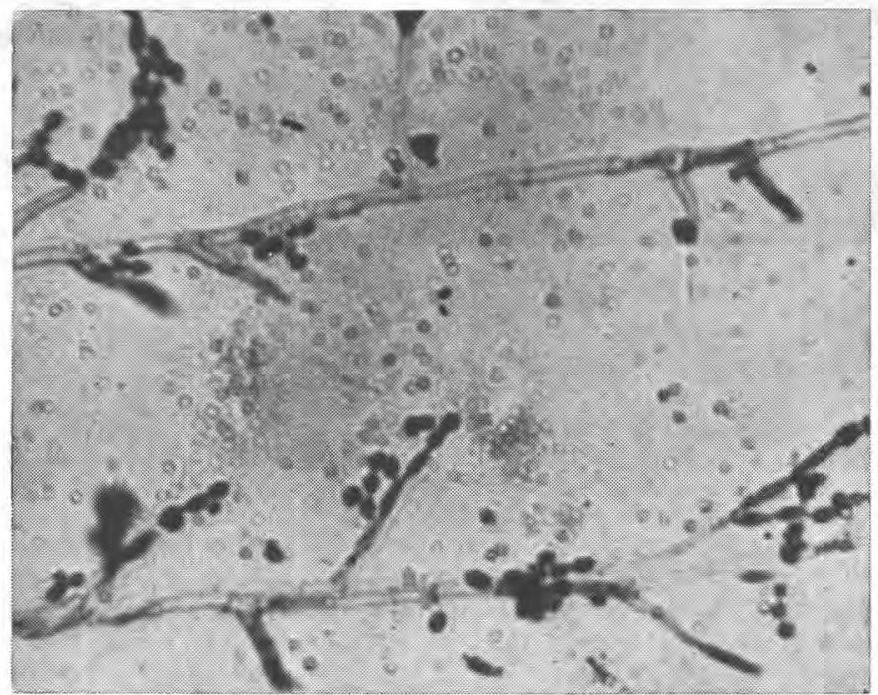

FIG. 2. - Microphotographie d'une culture de Cladosporium herbarum sur lactoserum. Les files de conidiospores extrêmement fragiles sont pour la plupart rompues, et les conidies parsèment toute la préparation. 

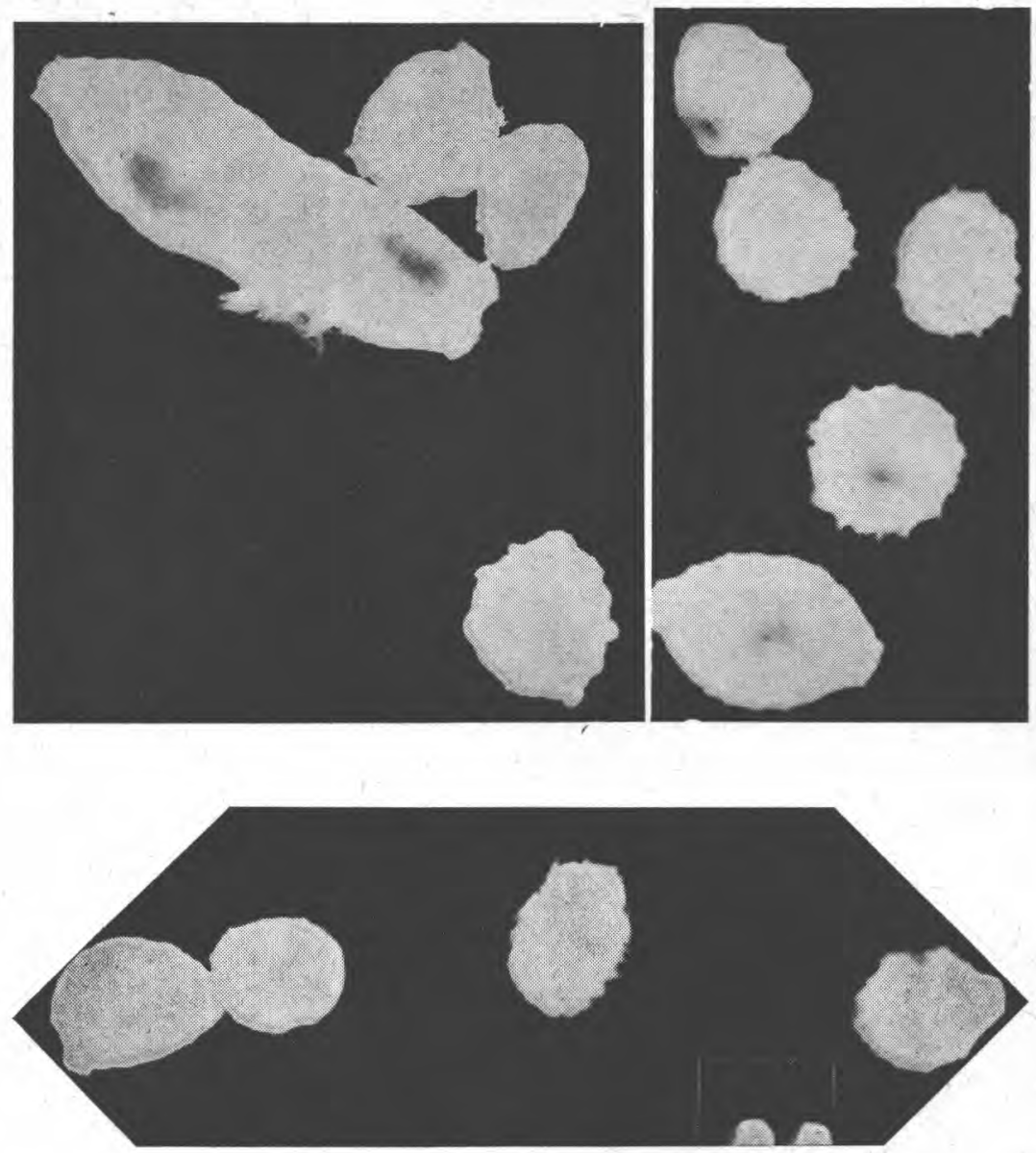

Fig. 3. - Aspect des conidies, légèrement échinées de Cladosporium herbarum au microscope électronique

Noter la forme allongée de l'article de base des files qui, en fait, est double (en haut à gauche), muni d'une cloison transversale dont on devine seulement ici l'emplacement. Les tailles et formes des spores sont variables selon leur position dans la chaîne conidienne, les terminales étant plus petites et rondes, les plus proches de la basale, plus grandes, plus épaisses, ovalaires, presque en citron.

On aperçoit bien, sur la spore de droite de la photographie du bas, par exemple, la partie plane qui la rattachait à l'élément suivant et par où se fera la germination du thalle.

(Les deux barres verticales du trait inférieur à droite de la même image limitent une distance de $1 \mu$, toutes les photographies étant prises à la même échelle.) 
herbarum dans laquelle il faut inclure toutes les formes vertes saprophytes communes. On tend, par ailleurs, à réserver le nom d'Hormodendron aux variétés robustes et ramifiées des Cladosporium (Langeron). De Vries [21] ne partage pas totalement cet avis, et, selon lui, le $C$. herbarum, parmi les autres membres du genre, se caractérise par :

- Un mycélium gris-vert, jamais orange ;

- Un revers des colonies vert-noir ;

- Des conidiophores noueux avec des prolongations distinctes; cellules ;

- Des conidies souvent verruqueuses, formées de une à quatre

- Des cellules d'un diamètre ordinaire de 4-5 $\mu$;

- Des conidies bi-cellulaires de 3 à $8 \mu$;

\section{III. - Description}

\section{A) Aspect morphologique.}

Nous avons isolé plusieurs fois des $C$. herbarum à partir de camemberts. Ils se ressemblaient tous suffisamment pour que nous n'ayons besoin d'en décrire qu'un seul.

Sur milieu de Czapeck, la germination d'une spore donne naissance à des filaments très ramifiés. Les hyphes paraissent plates, d'une largeur de 2 à $3 \mu$, d'une longueur de 150 à $300 \mu$ pour les parties aériennes. Les premiers articles ont de 50 à $90 \mu$; dans d'autres parties, 30 à $40 \mu$ et, parfois, on en observe de très courts : une dizaine de $\mu$; leur longueur est donc très variable, avec une largeur de 2 à $3 \mu$. Toutes ces parties sont plus ou moins rugueuses, cloisonnées, très colorées en vert brun foncé, comme les conidies, d'ailleurs.

Sur ces éléments, naissent des cellules ovales, généralement divisées en deux parties égales par une cloison, longues de 8 à $11 \mu$, d'un diamètre de 3 à $3,5 \mu$; parfois, bien que de mêmes dimensions, elles sont uni-cellulaires. Sur ces chaînes sont branchées d'autres files de spores qui les prolongent ou sont divergentes et partent alors près du sommet. Elles sont de grandeur variables, d'autant plus petites que plus jeunes et unicellulaires. Elles ont une longueur de 4 à $5,5 \mu$, avec un diamètre de $2 \mu$, mais plus généralement, de 3 à $3,5 \mu$. En vieillissant, les chaînes s'emmêlent et apparaissent en têtes denses, en forme d'arbre.

Les spores sont lisses; par coloration de contraste, nous en voyons quelques-unes finement échinées, ce que confirme le microscope électronique. Elles sont souvent réunies par groupe de deux. Certaines présentent une ou plusieurs cicatrices à l'endroit où elles étaient réunies aux autres (fig. 3 ). 
C. herbarum est fragile et se casse facilement au montage, les tiges souvent en fragments courts, tandis que les spores se détachent (fig. 2).

\section{B) Aspect des cultures.}

1. Sur milieu de Czapeck, la colonie apparaît le $3^{\mathrm{e}}$ jour après l'ensemencement, à la température du laboratoire. Elle est blanchâtre, mais vire au vert grisâtre, puis au jaune verdâtre foncé, pour finir en vert jaunâtre très foncé. Le centre est légèrement plus élevé, de couleur plus claire, d'aspect laineux, alors que le reste de la colonie est d'un fin velouté ras. La marge de la colonie est d'un blanc verdâtre. Les bords sont de couleur plus claire. Ils ne paraissent pas nets, mais finement découpés, par suite d'une croissance rayonnée. On n'aperçoit ni exsudat, ni zone, ni rayons. La colonie atteint un diamètre d'environ $7 \mathrm{~mm}$ le $5^{\mathrm{e}}$ jour, $17 \mathrm{~mm}$ le $10^{\mathrm{e}}$ jour et $2 \mathrm{~cm}$ le $13^{\mathrm{e}}$ jour après l'ensemencement.

Son revers, d'abord de couleur vert foncé, tourne au vert noir intense avec une légère opalescence. Le mycélium très foncé se distingue parfaitement quand le milieu est épais et forme un enchevêtrement ressemblant à de la mousse, quelquefois plus long que la partie aérienne elle-même. Les parties qui dépassent cet ensemble donnent par la ramification des branches, l'impression d'une tête de sapin.

2. Sur milieu de Czapeck peptoné, la croissance est plus rapide. La colonie apparaît le deuxième jour après l'ensemencement; on note un diamètre de $1 \mathrm{~cm}$ au $5^{\mathrm{e}}$ jour ; 2 au $10^{\mathrm{e}}$ et $3 \mathrm{~cm}$ au $15^{\mathrm{e}}$ jour. Elle croît avec un bord blanchâtre très net. Il y a une tendance tout juste marquée à la zonation. Le revers possède, lui aussi, une marge blanchâtre. On y distingue une ligne de zonation à la limite de la colonie, beaucoup moins épaisse. Par ailleurs, la colonie reproduit l'aspect décrit précédemment.

3. Sur malt peptoné glucosé, la colonie apparaît tardivement, vers la fin du $4^{\mathrm{e}}$ jour et présente un diamètre de $4 \mathrm{~mm}$ le $5^{\mathrm{e}}$ jour ; $14 \mathrm{~mm}$ le $10^{\mathrm{e}}$ et $2 \mathrm{~cm}$ vers le $15^{\mathrm{e}}$ jour. La colonie devient vert jaunâtre foncé, avec une bordure d'un millimètre plus blanchâtre. La zone centrale est plus touffue, par suite de la présence d'hyphes qui dépassent le niveau général; la partie externe est veloutée. Cette marge blanchâtre d'un millimètre se retrouve au revers. Pour le reste, l'aspect de la colonie est le même que sur milieu de Czapeck. 


\section{IV. - Moyens de Iutte contre Cladosporium herbarum}

Le $C$. herbarum est très commun et largement répandu, même dans l'air. Il pousse sur les matériaux cellulosiques, donc le papier, les textiles et également les peintures et vernis, le caoutchouc, le cuir et, naturellement, toutes sortes de nourritures et denrées. Il est très abondant sur de nombreux végétaux en décomposition et sur le foin. Il peut causer des altérations du malt et du houblon. II s'installe sur les taches du limbe de très nombreuses plantes (Lagière [10]. L'un de nous l'a signalé jusque sur cette curieuse graminée qui a envahi, à une certaine époque, les présalés de la Manche [4], la spartine de Townsend.

Il croît dans une grande marge de température puisqu'on l'a trouvé infectant de la viande stockée au frigorifique à - $6^{\circ} \mathrm{C}$ [1]. Bref, on le rencontre fréquemment dans les bâtiments où il forme de petites taches brun-noirâtres sur les murs.

Il intervient puissamment dans le rouissage sur terre ou sur pré des plantes textiles (Buchmann et Bartmann [2]). Il s'y montre d'ailleurs, beaucoup plus actif contre les pectines qui constituent une sorte de ciment, de gelée intercellulaire, que contre la cellulose vraie des parois. D'après Penau [17], qui cite la forme Hormodendron, il pourrait servir dans la biosynthèse des stéroïdes et serait capable d'hydroxyler en position 15 la substance S de Reichstein pour donner la $15-\alpha$-hydroxy-substance $\mathrm{S}$.

Il n'est donc pas du tout extraordinaire que nous l'ayons trouvé sur les camemberts où il peut être apporté par le lait ou l'air. Nous avons remarqué que les infections survenaient surtout au printemps et à l'automne. Il est plutôt étonnant, étant donné son abondance dans la nature, qu'il n'ait pas encore été signalé et n'ait pas provoqué d'accidents plus fréquents et plus nombreux : le fromage ne lui est peut-être pas tellement favorable comme milieu de culture.

Sur les camemberts, le $C$. herbarum apparaît au haloir à partir du $9^{\mathrm{e}}$ jour ou les jours suivants. D'abord, de petites taches jaunâtres se forment, mais cette phase passe souvent inaperçue, car la couleur vire très rapidement au vert, puis, au bout de quelques jours, au gris. Elles restent, généralement, peu étendues, tout au moins au début de l'infection et se localisent aux endroits où le $P$. caseicolum a peu poussé ou manque totalement, c'est-à-dire, sur les bords du fromage et dans les sillons laissés par l'empreinte des claies.

La rapidité de la contamination est apparemment surprenante. Nous avons vu des fromages parfaitement blancs le soir, être complètement tachés le lendemain, ou encore parfaits à l'emballage, arriver chez le destinataire contaminés.

Le $C$. herbarum donne, souvent, une odeur qui rappelle le champignon ou l'herbe fraîchement coupée. Nous avons souligné, ci- 
dessus, sa fragilité ; il "poudre " ainsi très facilement, ce qui augmente sa dispersion dans l'air. Cette remarque est, d'ailleurs, mise à profit par les fromagers qui frappent les camemberts atteints avant de les emballer, ce qui, effectivement, fait disparaître les taches des fromages anormaux, mais disperse l'infection et augmente considérablement l'ensemencement par l'ambiance et contribue à la généralisation de la contamination par le nuage de conidies qui - s'élève des produits ainsi traités.

Nous avons combattu, en 1955, le $C$. herbarum par les moyens habituels, e'est-à-dire, qu'en plus des soins de propreté et de nettoyage, nous avions cherché à obtenir la couverture du caillé la plus rapide et la meilleure possibles par le $P$. caseicolum. Les souches provenant directement de Neufchâtel, qui appartiennent à la même espèce botanique, donnent un feutrage épais, qui se développe rapidement. Elles étaient tout indiquées ici et produisirent de bons résultats. Les fabricants prirent, d'ailleurs, l'habitude de broyer des bondons dans le lait avant emprésurage, pour apporter très tôt les variétés vigoureuses de $P$. caseicolum du Neufchâtel qui combattaient l'infection. Mais, le goût du camembert s'en ressentait. Aussi, certains ne dispersaient-ils que la croûte de la bonde dans le lait. Nous remarquâmes que, dans ce cas, le remède paraissait moins efficace.

Lors de notre premier isolement, en étudiant le $C$. herbarum par culture sur différents milieux, nous arrivâmes à la conclusion que, s'il poussait bien en zone alcaline ou neutre, il ne se développait pratiquement pas au $p H 4,5$, qui est celui constaté généralement à la surface des camemberts au moment du démoulage. Cela nous fournissait une des explications de son apparition uniquement tardive, sur des surfaces rendues moins acides par le $P$. caseicolum, qui a déjà brûlé dans des combustions respiratoires une partie de l'acide produit antérieurement par les ferments lactiques.

Pour retarder cette neutralisation, nous préconisâmes done une bonne fermentation lactique. En fait, dans ce cas, l'apparition du $C$. herbarum se trouve reculée de quelques jours, ce qui, en permettant une meilleure pousse des appareils conidiens du Penicillium (" la fleur " des fromages) contribue à la disparition de la moisissure indésirable.

Pourtant, avec l'admirable plasticité des moisissures, nos derniers isolements nous montrent qu'actuellement le $C$. herbarum s'est adapté à des milieux plus acides qu'il y a dix ans. Nous en voulons comme preuve son développement, bien que tardif, sur milieu au malt peptoné glucosé qui, sauf son acidité, lui est, par ailleurs, très favorable.

L'observation allait, par ailleurs, nous mettre sur la voie d'un moyen beaucoup plus efficace de lutte.

Une fromagerie dont nous nous occupions avait un haloir qui 
ne servait qu'exceptionnellement, car il avait la réputation de donner le "bleu ». La période de grande production étant arrivée, il a bien fallu se résigner, quand même, à utiliser cette salle. La plupart des camemberts d'une journée de fabrication furent placés dans les locaux habituels et l'excédent dans le séchoir incriminé qui reçut, d'ailleurs, le lendemain, une partie de la fabrication suivante, le reste allant dans les haloirs normaux libérés. Il y avait, ainsi, des camemberts de deux journées de productions différentes, partiellement placées dans ce local ; comme prévu, ces fromages s'y couvrirent de taches, alors que les échantillons correspondants, servant en quelque sorte de témoins, logés dans les anciens locaux, restèrent parfaitement blancs.

Cette invasion massive éliminait toute possibilité de contamination autre que par la pièce elle-même. De plus, nous remarquâmes que si la fraction introduite la première était sérieusement infectée, la deuxième l'était beaucòup moins. Enfin, les contaminations affectaient surtout les fromages du haut et des côtés des étagères et beaucoup moins ceux situés en profondeur. De ces constatations, nous conclûmes que le conditionneur d'air, formé d'un radiateur sur lequel soufflait un ventilateur, devait être le responsable de la diffusion des spores. Les ailettes du radiateur, difficilement nettoyables, étaient, è fait, bourrées de spores de moisissures qui avaient le temps de sécher pendant la non utilisation du local et étaient entraînées, lors de la remise en route, par le courant d'air, sur les camemberts. Les plus proches (en haut et sur les côtés) en avaient récolté la majorité et les plus éloignés (en bas et au centre) le reste.

Dès lors, il fallait désinfecter le haloir préalablement vidé avec un antiseptique assez volatil pour pénétrer dans les moindres recoins et interstices du radiateur. Nous fîmes répandre plusieurs litres d'une solution de formol du commerce à 40 p. 100 , après avoir fait boucher les fentes des fenêtres et des portes par des bandes de papier collé, pour éviter toute communication avec les autres pièces. Ce haloir fut laissé ainsi pendant 24 heures, le ventilateur tournant, sans le froid. Puis, une autre journée fut nécessaire, pour l'aération destinée à chasser les traces de formol restant, toutes fenêtres ouvertes.

Cette désinfection étendue aux autres locaux et poursuivie systématiquement et périodiquement, supprima radicalement tous les accidents nommés "bleus" dans cette fromagerie, et ceci, malgré l'état vétuste de celle-ci.

Bien entendu, cette méthode est générale et permet une bonne désinfection des locaux en cas d'envahissement par des moisissures indésirables. Elle permet de lutter, efficacement, contre les contaminations par les Penicillia, que nous rencontrons maintenant, souvent associés au Cladosporium herbarum que nous trouvions. seul primitivement dans les accidents tardifs de " bleu ". 
On peut employer la solution de formol, soit en la laissant s'évaporer naturellement, soit en faisant dégager le formol par ébullition ou pulvérisation, où à l'aide d'un oxydant tel que le permanganate, ou encore en employant le trioxyméthylène. Nous avons quelquefois utilisé une solution d'ammoniaque pour détruire les dernières traces de formol, surtout si l'on ne dispose pas du temps nécessaire pour une aération suffisante. Le formol se combinę, en effet, avec l'ammoniac pour donner de l'hexaméthylènetétramine (ou hexamine).

Nous fîmes appliquer, par la suite, cette désinfection à d'autres fromageries avec toujours le même succès. Nous citerons simplement le cas de l'une d'elles où l'infection était telle que l'on en envisageait l'arrêt. La désinfection systématique et répétée rétablit rapidement la situation.

Malheureusement, il arrive, aussi, que la disposition des locaux empêche d'isoler certains d'entre eux pour les désinfecter. Il faut, alors, recourir à la première méthode citée, mais qui est bien moins efficace.

Il est bon, enfin, de pratiquer des analyses d'atmosphère en exposant des boîtes de Petri ouvertes et contenant un milieu de culture favorable, comme du lactosérum gélosé. On peut suivre, ainsi, la marche des opérations et se rendre compte que, parfois, une seule désinfection ne suffit pas, la contamination des locaux voisins (couloirs notamment) réensemençant l'atmosphère.

Le $C$. herbarum végète bien à basse température. Il ne faut donc pas compter sur le froid pour arrêter son développement. Le $P$. caseicolum, en revanche, y est beaucoup plus sensible.

(Laboratoire de Microbiologie de la Faculté des Sciences de Caen, Centre de 3 e Cycle de Sciences du Lait et Centre du Lait et des Viandes de l'Université de Caen).

\section{BIBLIOGRAPHIE}

[1] F. BRooks et C. HAMSFORD. - Mould growths upon cold-store meat. Trans. Brit. Myc. Soc., 1922, 8, 113-142.

[2] Buchsmann et Bartram. - Zentr. $f$. Bakter., 1940, 2, 102, 300-302 et $365-387$.

[3] M. Desfle urs. - Contribution à la connaissance du genre Penicillium. Application à la fromagerie de pâtes molles et notamment du camembert. Thèse de doctorat de spécialité (Sciences du Lait), Caen, 1966.

[4] J. JacQUet. - Recherches écologiques sur le littoral de la Manche. Les présalés et la spartine de Townsend. Les estuaires. La tangue. 1949, I vol. Lechevalier, éd., Paris. 
[5] J. JAcquet. - Les moyens de lutte contre l'accident du "bleu " en fromagerie de pâtes molles du type camembert. Rôle des ferments. C.R.Ac.Sc., 1956, 243, 1570-1571.

[6] J. JACQUET. - Sur les moyens de lutte contre l'accident du "bleu" en fromagerie de pâtes molles du type camembert. Influence du milieu, notamment rôle du sel. C.R.Acad. Agric., 1957, 43, 129-132.

[7] J. JACQUET. - Les maladies des fromages, simple curiosité ou nouvel aspect de la pathologie comparée. Rev. Path. Gén. et Physiol. Clin., 1963, 63, 1007-1044.

[8] J. JacQUet et F. Niderprim. - Influence des impuretés du sel sur la fabrication du camembert. Bull. Soc. Chim. Fr., 1951, no 5-6, 310.

[9] J. Jacquet, O. Villette (Mme), J. Delacroix, H. Gondouin et M. Desfleurs. - Considérations sur l'action du $p \mathrm{H}$ dans la croissance des moisissures utilisées pour la fabrication du camembert. Rôle du sel. Bull. Soc. Linn. Norm., 1954, 9e sér., 8, 115-133.

[10] R. LAGIÈre. - Etude de quelques Cladosporium. Ann. Grignon, 1946, 3e sér., 5, 147-160.

[11] M. Langeron. - Précis de mycologie. 1 vol., Masson, éd. Paris, \1945.

[12] E. MASON. - Annotáted account of fungi received at the Imperial myeological Institute, List II, fase. 3, 1937, Publication de l'Institut Imperial de Mycologie, Kew.

[13] E. Mason. - List II, fase. III, General part. Ibid., 1937.

[14] E. MASon. - List II, fase. III, Special part. Ibid., 1941.

[15] A. Masson. - Contribution à l'étude de l'amélioration des sels de fromagerie. Diplôme d'Etudes Supérieures de la Faculté des Sciences de Nancy et Bull. Soc. Sc. Nancy, 1959.

[16] R. Menderez. - Etude de l'action du sel sur le développement du Penicillium candidum. Diplôme d'Etudes Supérieures de la Faculté des Sciences de Nancy et Bull. Soc. Sc. Nancy, 1958.

[17] H. Penau. - Fermentation mierobienne et biosynthèse chimique des médicaments. Tournées Pharm. Fr., 1 vol., 1957. Sepes, éd., Paris.

[18] P. SACCARDo. - Conspectus generum fungorum Italiae inferiorum nempe ad Sphaeropsideas, Melanconieas et Hyphomycetas pertinentium, systemate sporologico dispositorum. Mirhelia, 1880, 2, 1-38.

[19] P. SACCARDo. - Sylloge fungorum omnium hucusque cognitorum, 1884, 3, Pavie.

[20] P. SaCCARDo. - Ibid., 1886, 4.

[21] G. DE VnIEs. - Contribution to the knowledge of the genus Clados.porium, Link ex Fries, 1 vol., 1952, Uitgeverij et Drukkerij Edit. Baarn, 